\title{
ISE ARRAYS WITH IMPROVED DYNAMIC RESPONSE AND LIFETIME
}

\author{
Steven M. Martin ${ }^{1}$, Jeonghan $\mathrm{Ha}^{2}$, Jin Wook Kim ${ }^{2}$, Timothy D. Strong ${ }^{1}$, \\ Geun Sig Cha ${ }^{2}$, Richard B. Brown ${ }^{1}$
}

\author{
${ }^{1}$ Dept. of Electrical Eng. and Computer Science, \\ University of Michigan, Ann Arbor, MI USA
}

\author{
${ }^{2}$ Dept. of Chemistry, Kwangwoon University, \\ Nowon-gu, Seoul, South Korea
}

\begin{abstract}
Poly(vinlychloride) (PVC) based ion-selective electrodes (ISEs) exhibit poor substrate adhesion and often fail due electrolytic shunts around the membrane. Silicone rubber (SR) based ISEs have improved surface adhesion, solving the shunt problem, but they have over $15 \mathrm{x}$ larger impedances. This increased impedance yields noisy signals and long response times. An ion-selective electrode array with on-chip CMOS buffers was implemented to negate the effects of the silicone rubber membrane's increased impedance. The array was implemented using post-CMOS thin-film fabrication techniques. Theoretical calculations for ISE response time are presented and experimentally verified. Results demonstrated that the dynamic response of on-chip buffered ISEs was $7.5 \mathrm{x}$ faster than conventional electrodes, even though they were 225 times smaller. The silicone rubber ISEs demonstrated a 40x increase in lifetime versus PVC membranes, functioning properly for more than 120days.
\end{abstract}

\section{INTRODUCTION}

Poly(vinylchloride) (PVC) is a commonly used membrane in ion-selective electrodes (ISEs) due to its superb electrochemical properties. PVC, however, has poor mechanical adhesion to the silicon substrates often used in micropotentiometric sensors [1]. Poor adhesion is the leading cause of failure in solid-state potentiometric sensors [2]. Membrane matrices having better mechanical properties have been previously investigated. Of these, silicone rubber (SR) has shown exceptional adhesive strength to silicon nitride substrates [3-5].

Compared to PVC, silicone rubber membranes have larger source impedances. Conventional ion-selective electrodes can utilize silicone rubber membranes because the ISEs have large surface areas, and impedance is inversely proportional to area [6]. Solidstate ISE arrays, however, use small geometries which results in ISEs having large impedances, slow responses, and poor detection limits [5]. Membrane impedance may be reduced by engineering the membrane composition. Adding lipophilic salts and various plasticizers can reduce impedance, but they also reduce adhesion [4-5].

In this work, the impedance, response, and lifetime of several silicone rubber membranes were investigated. A solid-state ISE array with on-chip CMOS buffers was developed to enable the use of silicone rubber micropotentiometric sensors. The array was fabricated using a commercial CMOS process and the electrodes and membranes were post-processed on top of the electronics. The membranes were characterized on three electrodes including: conventional liquid junction, passive solid-state, and the CMOS-inte-

Travel support has been generously provided by the

Transducers Research Foundation and by the DARPA

MEMS and DARPA BioFlips programs.

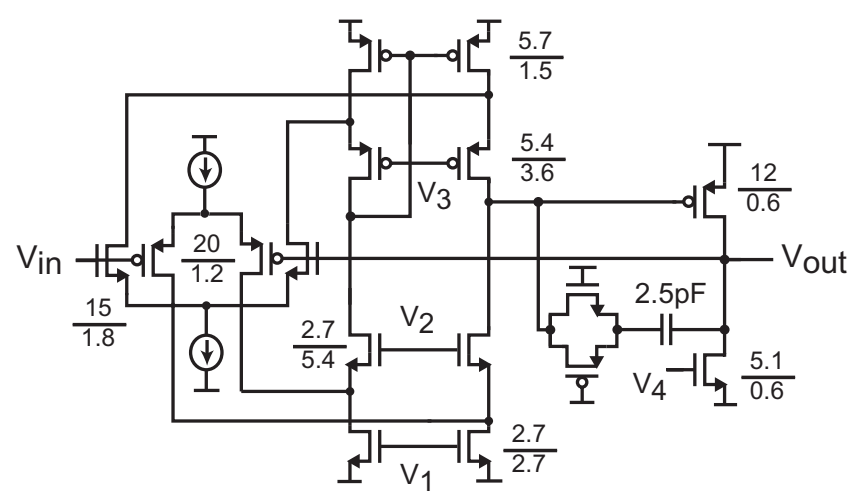

Figure 1. Unity-gain buffer.

Table I. Measured unity-gain buffer characteristics.

\begin{tabular}{lll}
\hline Specification & Conditions & Value \\
\hline Supply & $\mathrm{R}_{\text {load }}=1 \mathrm{M} \Omega$ & $0.9 \mathrm{~mW}$ \\
Power & Simulated & $100 \mathrm{~dB}$ \\
Open-loop gain & $\mathrm{R}_{\text {load }}=1 \mathrm{M} \Omega$ & $-0.98 \mathrm{~V} / \mathrm{V}$ \\
Closed-loop gain & $\mathrm{C}_{\text {load }}=13 \mathrm{pF}$ & $2.1 \mathrm{MHz}$ \\
3dB Bandwidth & Freq $=0 \mathrm{~Hz}$ & $>20 \mathrm{G} \Omega$ \\
Input Impedance & & $<100 \Omega$ \\
Output Impedance & & $2.6 \mathrm{~V}$ \\
Input Common Mode Range & & $2.6 \mathrm{~V}$ \\
Output Swing & $\mathrm{R}_{\text {load }}=1 \mathrm{M} \Omega$ & $20 \mathrm{mV} / \mu \mathrm{s}$ \\
Slew Rate & $\mathrm{C}_{\text {load }}=13 \mathrm{pF}$ & $3.5 \mu \mathrm{s}$ \\
Settle Time (1 V step) & $\mathrm{C}_{\text {load }}=13 \mathrm{pF}$ & $0.023 \mathrm{~mm}^{2}$ \\
Area & & \\
\hline
\end{tabular}

grated solid-state electrodes. A model of the ISE response was developed and experimentally verified. Lifetime of the various devices was also examined.

\section{EXPERIMENT}

Arrays of CMOS op-amps, configured as unity-gain buffers, were fabricated in an AMI Semiconductor (Pocatello, ID) $0.5 \mu \mathrm{m}$, $3 \mathrm{~V}$, CMOS process. The folded-cascode op-amps incorporated railto-rail input and output stages. Figure 1 shows the schematic of the buffers. Table I lists the measured performance of the unity-gain buffer.

Each die was designed to have six ion-selective electrodes with a $400 \mu \mathrm{m}$ diameter membrane and a $200 \mu \mathrm{m}$ diameter $\mathrm{Ag} / \mathrm{AgCl}$ electrode. The electrode arrays were fabricated on top of the active die using CMOS-compatible, thin-film processing techniques. Fig- 


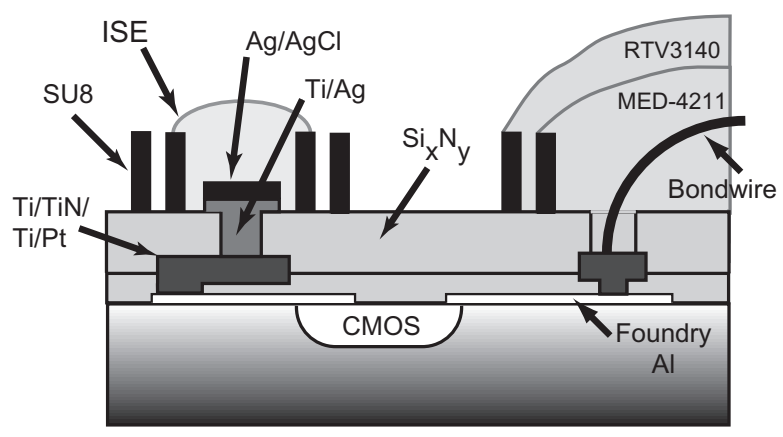

Figure 2. Cross-section of an active ISE.

ure 2 shows a cross-section of the completed devices. After delivery from the foundry, the CMOS devices were patterned for interconnect deposition and subjected to a short reactive-ion etch (RIE) to improve step coverage and patterning. A layer of Ti/TiN $(40 / 80 \mathrm{~nm})$ was sputter-deposited to prevent interdiffusion between the aluminum and platinum layers. A layer of Ti/Pt (10/1000nm) was then evaporated onto the die and patterned using liftoff. A passivation layer of $400^{\circ} \mathrm{C}$ PECVD silicon nitride (800nm) was then deposited. Contact openings through the nitride were etched using RIE. Ti/Ag (20/650nm) was then sputter deposited and patterned using liftoff. Thin wells $(12 \mu \mathrm{m}$ high) were patterned in SU-8. The SU- 8 was cured at $150^{\circ} \mathrm{C}$ and became a permanent feature on the die. Next, the arrays were diced and bonded. The wirebonds were covered with a two step polymer coating of a silicone elastomer (MED-4211, NuSil Silicon Technology, Santa Barbara, CA) and silicone rubber (RTV3140, Dow Chemical, Midland, MI). Finally, the $\mathrm{Ag}$ electrodes were chloridized by submersion in $1 \mathrm{M} \mathrm{FeCl}_{3}$. Passive electrode arrays of identical size were similarly fabricated.

Three different silicon rubber (RTV3140) potassium-selective membranes (labeled K1-K3), and a PVC potassium-selective membrane (K4) were made according to Table II. The common additive potassium tetrakis[3,5-bis(p-chlorophenyl)borate (KTp$\mathrm{ClPB}$ ) and plasticizer dioctylsebacate (DOS) were incorporated into the membranes to study their effects on altering the membrane impedance and performance. The membranes were dissolved in tetrahydrofuran (THF) and dispensed into the SU-8 rings. The rings ensure a repeatable membrane thickness $(\approx 12 \mu \mathrm{m}$ thick). The solvent was allowed to evaporate over several days, leaving behind the selective polymer membrane. Microphotographs of completed devices are shown in Figure 3.

\section{RESULTS AND DISCUSSION}

The impedance of the membranes on the different electrode types was recorded using electron impedance spectroscopy (EIS) in a solution of $50 \mathrm{mM}$ Tris- $\mathrm{H}_{2} \mathrm{SO}_{4}, \mathrm{pH} 7.4$ plus $0.1 \mathrm{M} \mathrm{KCl}$. The results of the EIS experiments were averaged over four different devices

Table II. Membrane compositions.

\begin{tabular}{lllll}
\hline Components & K1 & K2 & K3 & K4 \\
\hline Matrix & RTV3140 RTV3140 & RTV3140 PVC \\
Additive: KTpClPB & $0.6 \mathrm{mg}$ & & & \\
Plasticizer: DOS & & $13.3 \mathrm{mg}$ & & $133 \mathrm{mg}$ \\
Ionophore: valinomycin 2.0 mg & $2.0 \mathrm{mg}$ & $2.0 \mathrm{mg}$ & $2.0 \mathrm{mg}$ \\
\hline
\end{tabular}

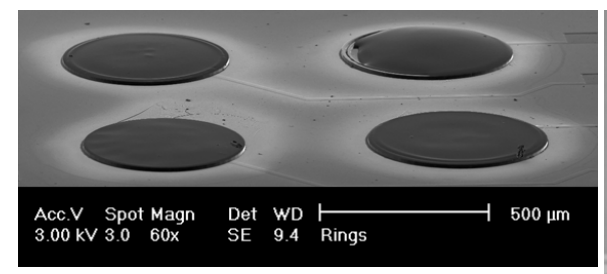

(a)

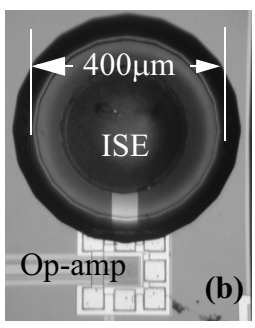

Figure 3. (a) Scanning electron micrograph of four ISEs cast into the SU-8 containment rings. (b) Microphotograph of an active ISE showing the buffer op-amp and selective membrane.

Table III. Measured membrane impedances in $M \Omega$

\begin{tabular}{llllll}
\hline Electrode Type & $\begin{array}{l}\text { Area } \\
\left(\mathrm{mm}^{2}\right)\end{array}$ & $\mathrm{K} 1$ & $\mathrm{~K} 2$ & $\mathrm{~K} 3$ & $\mathrm{~K} 4$ \\
\hline $\begin{array}{l}\text { Conventional } \\
\text { Solid-State (pas- }\end{array}$ & 0.13 & 555 & 127 & 601 & \\
\begin{tabular}{l} 
sive) \\
\hline
\end{tabular}
\end{tabular}

and are shown in Table III. The impedance of the active devices could not be measured because of the on-chip buffer, but their impedance should match that of the passive devices. The passive electrodes had impedances well into the gigaohm range even with the various additives. Comparatively, the average impedance of the PVC-based membrane was $307 \mathrm{M} \Omega$. These results demonstrate that the silicone rubber membranes were nearly $15 x$ higher resistance.

The selectivity of the membranes was characterized using the matched potential method [7], and the results are shown in Table IV. The selectivity coefficient is a measure of the ratio of primary to interfering ion activity that results in an identical potential change of the sensor. The results indicate that the potassium-selective silicone rubber membranes are over four orders of magnitude more selective towards potassium than the most of other ions examined. The selectivity of the membranes is affected by the addition of the lipophilic salt. The results show that the selectivity is compromised the most in the lowest impedance silicone rubber membrane, K2.

The dynamic response of an ISE can be simulated using a two-time-constant model. The solution/membrane system has an intrinsic time constant, $\tau_{1}$ [8]. The corresponding dynamic potential change of the membrane is given by

$$
\Delta V_{\text {mem }}(t)=\frac{R T}{z F} \ln \left(\frac{a_{f}}{a_{i}}\right)\left[1-\exp \left(-\frac{t}{\tau_{1}}\right)\right]
$$

where $t$ is time, $R$ is the gas constant, $T$ is temperature, $z$ is the charge number of the ion, $F$ is Faraday's constant, $a_{f}$ is the final activity of the ion, and $a_{i}$ is the initial activity of the ion. A second time-constant, $\tau_{2}$, is formed by the membrane impedance in series with the input capacitance of the measuring circuit, $\mathrm{C}_{\text {load }}$, as shown in Figure 4. Assuming an instantaneous change in activity, the change in output voltage can be derived from Figure 4 such that

$$
\Delta V_{\text {out }}(t)=\frac{R T}{z F} \ln \left(\frac{a_{f}}{a_{i}}\right)\left[1+\frac{\tau_{2} \exp \left(-\frac{t}{\tau_{2}}\right)}{\tau_{1}-\tau_{2}}-\frac{\tau_{1} \exp \left(-\frac{t}{\tau_{1}}\right)}{\tau_{1}-\tau_{2}}\right] .
$$

Note that the effective impedance of the reference electrode is negligible compared to the resistance of the membrane. A commercial 
Table IV. Measured selectivity coefficients.

\begin{tabular}{lllll}
\hline Selectivity $\log \left(\mathrm{K}_{\mathrm{k}, \mathrm{j}}\right)$ & $\mathrm{Na}^{+}$ & $\mathrm{Ca}^{2+}$ & $\mathrm{Mg}^{2+}$ & $\mathrm{NH}_{4}^{+}$ \\
\hline K1 Membrane & $<-5.0$ & $<-5.0$ & -4.2 & -2.0 \\
K2 Membrane & -4.8 & -4.6 & -3.9 & -1.9 \\
K3 Membrane & $<-5.0$ & $<-5.0$ & -4.1 & -2.0 \\
\hline
\end{tabular}

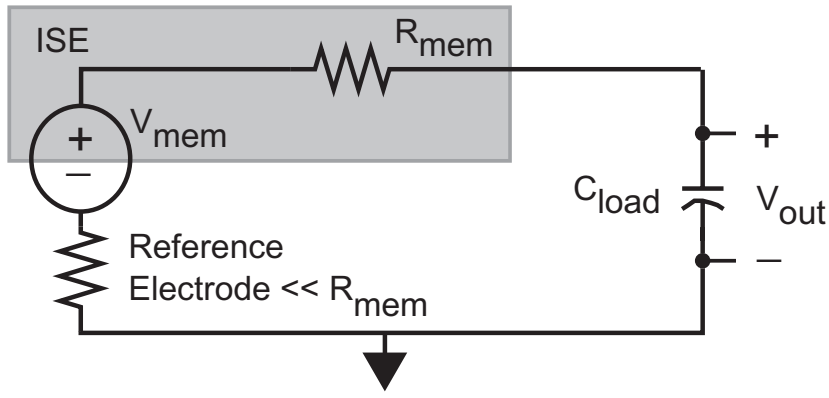

Figure 4. Simple model for ISE dynamic response.

15-channel pH meter (Kosentech, South Korea), with a measured input capacitance of $1 \mathrm{nF}$, was used for recording. The active devices' input capacitance was approximately 100fF. Table V lists the corresponding time constants. Since the active devices minimize $\tau_{2}, \tau_{1}$ was empirically fit using the active devices' response. Figure 5 shows both the theoretical and experimentally measured dynamic responses to a decade change in concentration of $\mathrm{K}^{+}$. The experiments were conducted in a solution of $50 \mathrm{mM}$ Tris- $\mathrm{H}_{2} \mathrm{SO}_{4}$, $\mathrm{pH}$ 7.4. Voltage offsets of the responses were eliminated mathematically before plotting. The figure clearly demonstrates the response time dependence on the membrane impedance and load capacitance. The active ISEs responded, on average, nearly 200x faster than passive ion-selective electrodes. The CMOS-buffered ISEs, which are $225 \mathrm{x}$ smaller than the conventional electrodes, were $7.5 \mathrm{x}$ faster than the conventional electrodes.

Figure 6 shows the measured calibration curves for the potassium-selective electrodes in a solution of $50 \mathrm{mM}$ Tris- $\mathrm{H}_{2} \mathrm{SO}_{4}, \mathrm{pH}$ 7.4. At room temperature, the theoretically calculated slope for potassium-selective electrodes is $59 \mathrm{mV} / \mathrm{dec}$. The percent error of the measured slope versus this ideal slope was $5 \%$ and $19 \%$ for the active and passive devices, respectively. The standard deviation of the slope was $0.7 \mathrm{mV} / \mathrm{dec}$ for the active ISEs while the standard deviation of the slope was $3.0 \mathrm{mV} / \mathrm{dec}$ for the passive sensors.

Figure 7 plots the slope of the calibration curve versus soaking time. The PVC membrane failed after 3days in $50 \mathrm{mM}$ Tris$\mathrm{H}_{2} \mathrm{SO}_{4}, \mathrm{pH} 7.4$, while the passive silicone rubber membrane survived nearly 50days before unexpectedly failing. All six of the passive ISEs failed on the same day indicating some catastrophic failure on the die. This failure is most likely unrelated to failures in

Table V. Measured time constants in ms.

\begin{tabular}{lcc|cc|cc}
\hline Electrode Type & \multicolumn{2}{c|}{ K1 } & \multicolumn{2}{c|}{ K2 } & \multicolumn{2}{c}{ K3 } \\
& $\tau_{1}$ & $\tau_{2}$ & $\tau_{1}$ & $\tau_{2}$ & $\tau_{1}$ & $\tau_{2}$ \\
\hline Conventional & 0.9 & 560 & 0.5 & 130 & 0.8 & 600 \\
Solid-State (passive) & 0.9 & 8800 & 0.5 & 4600 & 0.8 & 8000 \\
Solid-State (active) & 0.9 & 0.9 & 0.5 & 0.5 & 0.8 & 0.8 \\
\hline
\end{tabular}

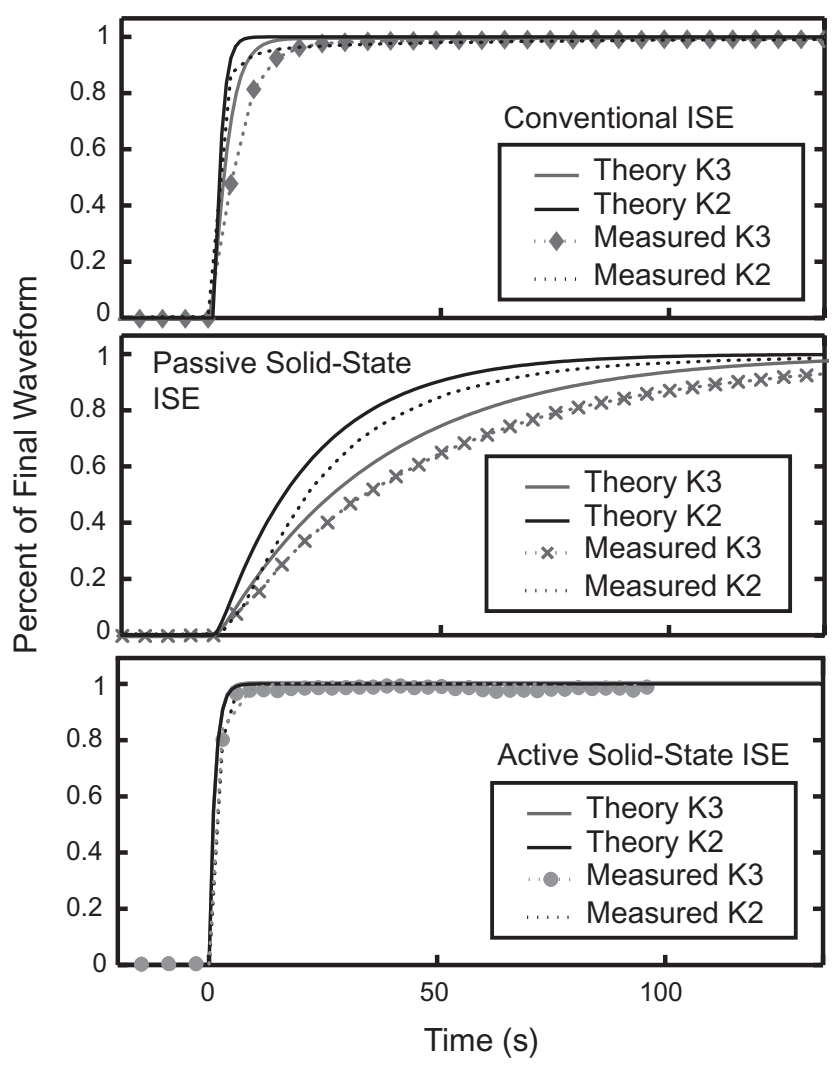

Figure 5. Theoretical and measured dynamic response for the $K 2$ and $K 3$ membranes on the three electrode types.

the ion-selective electrodes themselves. The active ISEs with silicone rubber membranes functioned properly for more than 120days.

\section{CONCLUSION}

The measured characteristics of the ion-selective electrodes are summarized in Table VI. The impedance of the silicone rubber membranes was shown to be $15 \mathrm{x}$ more resistive than PVC membranes. Incorporation of additives such as lipophilic salts into the membrane were unsuccessful at lowering the impedance below $1 \mathrm{G} \Omega$ CMOS-integrated ISE arrays were fabricated using thin-film post-processing techniques to enable the use of the high-resistance

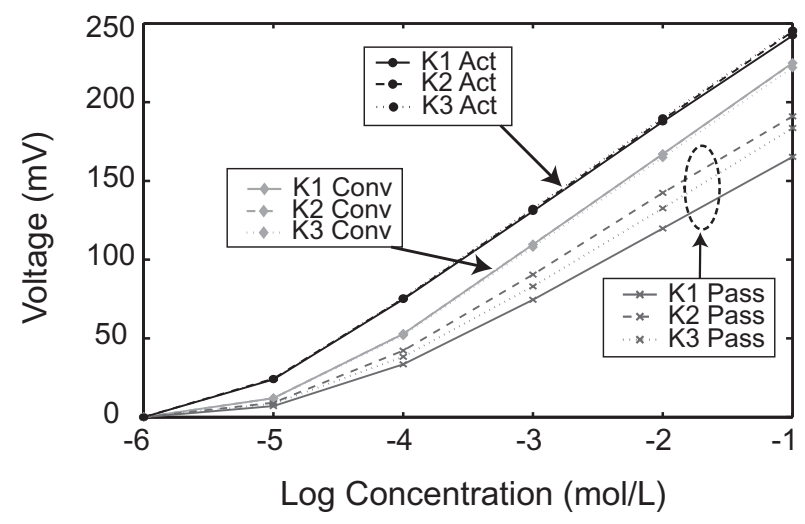

Figure 6. Measure calibration curves for the ISEs. 
Table VI. Summary of measured ISE characteristics.

\begin{tabular}{|c|c|c|c|c|c|c|}
\hline Membrane & Electrode Type & $\begin{array}{l}\text { Impedance } \\
(\mathrm{M} \Omega)\end{array}$ & $\begin{array}{l}\text { Slope } \\
(\mathrm{mV} / \mathrm{dec})\end{array}$ & $\begin{array}{l}\text { Response Time } 95 \% \\
\text { (s) }\end{array}$ & $\begin{array}{l}\text { Detection Limit } \\
(\mu \mathrm{M})\end{array}$ & $\begin{array}{l}\text { Lifetime } \\
\text { (days) }\end{array}$ \\
\hline & conventional & 555 & 56.0 & 5 & 14 & $>120$ \\
\hline \multirow[t]{3}{*}{$\mathrm{K} 1$} & passive & 8766 & 44.1 & 234 & 17 & 46 \\
\hline & active & & 56.5 & 1 & 9.4 & $>120$ \\
\hline & conventional & 127 & 56.0 & 12 & 17 & $>120$ \\
\hline \multirow[t]{3}{*}{$\mathrm{K} 2$} & passive & 4600 & 49.8 & 130 & 12 & 46 \\
\hline & active & & 55.9 & 1 & 10 & $>120$ \\
\hline & conventional & 601 & 56.5 & 22 & 15 & $>120$ \\
\hline \multirow[t]{2}{*}{$\mathrm{K} 3$} & passive & 7967 & 48.6 & 250 & 15 & 46 \\
\hline & active & & 55.0 & 3 & 9.9 & $>120$ \\
\hline
\end{tabular}

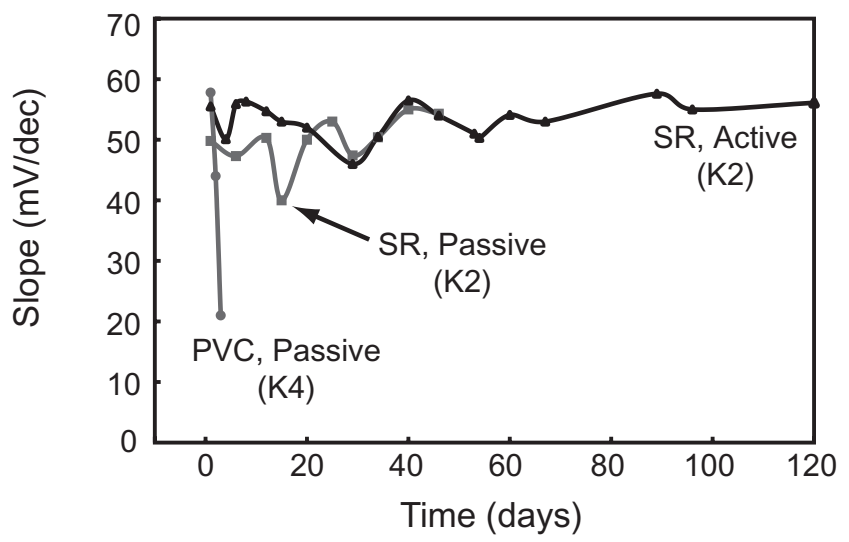

Figure 7. Measured lifetime of SR and PVC membranes.

silicone rubber membranes in micropotentiometric sensors. Results indicated that the buffered ISEs had a 200x faster response than passive potentiometric sensors. The active devices were even faster than conventional electrodes while occupying $225 \mathrm{x}$ smaller area. The active ISEs improved the slope and linearity of the response versus the passive sensors. The active, SR-based ISEs survived for more than 120 days with a response within $5 \%$ of ideal and a detection limit of $10 \mu \mathrm{M}$. Response times, detection limits, and lifetimes for the economically fabricated active sensors were far superior to those of conventional and passive microelectrodes.

\section{ACKNOWLEDGEMENTS}

This work was supported in part by the Engineering Research Centers Program of the US National Science Foundation under Award Number EEC-9986866 and under a National Science Foundation Graduate Research Fellowship. The authors would like to thank the MOSIS MEP research support program for fabrication of the CMOS electronics.

\section{REFERENCES}

[1] H. Nam, G.S. Cha, T.D. Strong, J. Ha, J.H. Sim, R.W. Hower, S.M. Martin, and R.B. Brown, "Micropotentiometric Sensors," Proceedings of the IEEE, vol. 91, pp. 870-880, June 2003.

[2] R.B. Brown, "An Integrated Multiple-Sensor Chemical Transducer," Ph.D. Dissertation, University of Utah, 1985.

[3] G.S. Cha, D. Liu, M.E. Meyerhoff, H. C. Cantor, A.R. Midgley, H.D. Goldberg, and R.B. Brown, "Electrochemical Performance, Biocompatibility, and Adhesion of New Polymer Matrices for Solid-State Ion Sensors," Analytical Chemistry, vol. 63, pp.1666-1670, 1991.

[4] I.J. Yoon, D.K. Lee, H. Nam, G.S. Cha, T.D. Strong, and R.B. Brown, "Ion sensors using one-component room temperature vulcanized silicone rubber matrices," Journal of Electroanalytical Chemistry, vol. 464, pp. 135-142, 1999.

[5] E. Malinowska, V. Oklejas, R.W. Hower, R.B. Brown, and M.E. Meyerhoff, "Enhanced electrochemical performance of solid-state ion sensors based on silicone rubber membranes," Sensors and Actuators B, vol. 33, pp. 161-167, 1996.

[6] A. Bard, and L. Faulkner, Electrochemical Methods: Fundamentals and Applications, John Wiley \& Sons, New York, NY, 1980.

[7] E. Baker, E. Pretsch, and P. Buhlmann, "Selectivity of Potentiometric Ion Sensors," Analytical Chemistry, vol. 72, pp. 11271133, 2000.

[8] W.E. Morf, E. Lindner, and W. Simon, "Theoretical Treatment of the Dynamic Response of lon-Selective Membrane Electrodes," Analytical Chemistry, vol. 47, pp. 1596-1601, 1975. 